\title{
Design and Implementation of Power Flow Calculation Service Based on WCF
}

\author{
Lifang $\mathrm{Xu}^{1, \mathrm{a}}$, Tong Jiang ${ }^{1, \mathrm{~b}}$, Zhao $\mathrm{Yu}^{1, \mathrm{c}}$ and Chengnan $\mathrm{Lu}^{1, \mathrm{~d}}$
}

${ }^{1}$ School of Electrical \& Electronic Engineering, North China Electric Power University,102206, China;

amaoxian_134@126.com, bjiangtong@ncepu.edu.cn, 557523096@qq.com, d614602585@qq.com

Keywords: Power flow calculation, WCF, SOA, Distributed Computation, Multithreading.

Abstract. Some problems exist in power flow calculation such as complex programming and expensive calculation software, a new method is put forward that traditional power flow calculation can be transferred to the Web. Based on that, this paper designs a power flow calculation service. The users can view the service interface and the instruction, add the service to the local program so as to complete the power flow calculation; besides, users can also access the relevant website through browser, upload raw data, and figure out power flow calculation easy and quickly. The method provides a clear and open power flow calculation service, which is convenient to use and can be applied to the teaching and authentication of computation results. In this paper, the Newton-Raphson power flow algorithm in Cartesian coordinates is demonstrated to verify the feasibility of the design.

\section{Introduction}

Power flow calculation is the basic calculation of power system steady-state operation, which can figure out voltage amplitude and phase angle of each node, power distribution and power loss of the network, thereby to determine the power system operating state, is of great significance for the studies of power system. Although the power flow calculation has been very mature, in practical application, due to complex programming and expensive calculation software, there are lots of inconveniences for beginners or ordinary users.

To solve this problem, this paper put forward a method that traditional power flow calculation can be transferred to the Web by WCF. In this paper, the WCF power flow calculation interface is compiled, which can realize the call of the WCF power flow calculation service, and the analysis of the calculation results. At the same time, it also provides an online platform for power flow calculation. The online platform adopts B/S architecture, based on WCF technology, to realize the multi-threading, distributed power flow calculation. The Newton-Raphson power flow algorithm in Cartesian coordinates is demonstrated to verify the feasibility of the design.

\section{Design of WCF Interface for Power Flow Calculation}

Newton-Raphson method is a typical method of solving nonlinear equations; the method has a square convergence property, which is widely used in steady-state power flow calculation. The WCF power flow calculation service established in this paper is based on the Cartesian coordinate system.

In this paper, the power flow calculation is divided into several functions, and each function is packaged as a WCF service, releasing by IIS (Internet Information Service), and waiting for clients to call. WCF power flow function interface and calling procedure as shown in Figure 1. In the process of calculation, the client can display the data structure of node and branch to view the corresponding data and calculation results. In the course of the call, the user can also compile the program by itself instead of calling interface program. By comparing the calculated results, the users can achieve the purpose of self-learning, and maximize the exchange of information. 


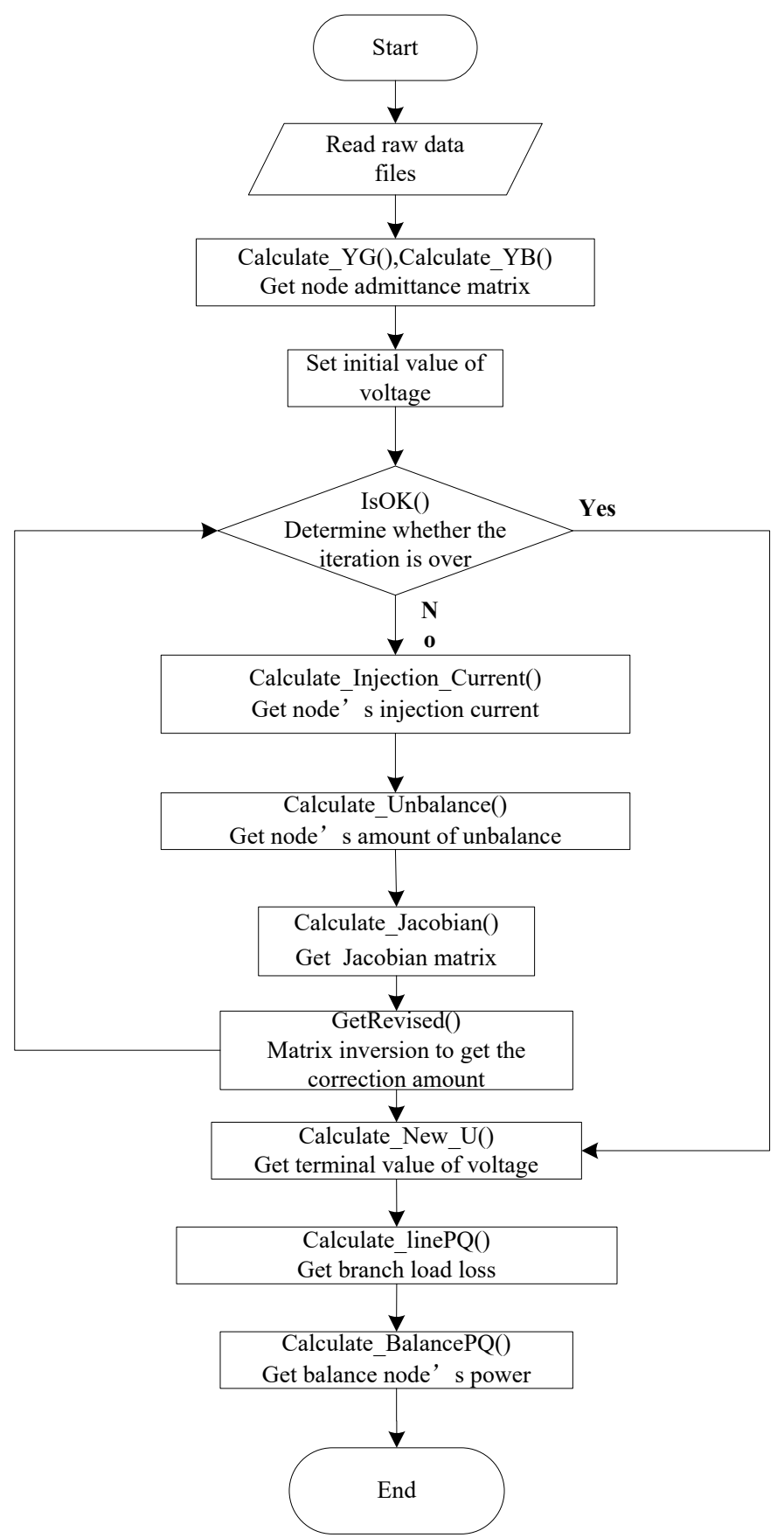

Fig. 1 WCF power flow function interface and calling procedure

\section{Framework Design for Power Flow Online Service Platform}

Power flow online service platform uses the service oriented architecture (SOA), and is divided into three subsystems, including user terminal subsystem, Web and WCF service management subsystem.

User Terminal Subsystem. User terminal subsystem consists of browsers. The user terminal subsystem is responsible for the data exchange between user and Web server and WCF server, and provides a friendly user interface to guide the user to conduct business. User terminal subsystem can be used to achieve the choice and upload of local raw data files, setting of calculate accuracy and download of calculation result by Asp.Net and Ajax.

Web Management Subsystem. Web management subsystem receives the request of the client and transfers it to the specified WCF server, and is responsible for realizing the load balance of the power flow calculation platform, which is fulfilled by the Web server. 
Web server couple with each WCF server through the network, Web server can get the working state and load rate of each WCF server, and provide decision support for load balancing.

Web management subsystem will be responsible for multi-threading management of power flow calculation; specific content will be introduced in the fourth chapter of this paper.

WCF Service Subsystem. WCF service subsystem consists of a number of WCF power flow calculation servers, is responsible for the calculation of the power flow. Power flow calculation servers use WCF as the basic framework; the business logic, data logic are encapsulated into WCF services, waiting for the call. When a client browser request transferred to the WCF server, the WCF server receives raw data files and carries out power flow calculation. After the calculation completed, the new value of voltage, branch power loss and balance node power are returned to the client in the form of DataSet database. Node admittance matrix and Jacobian matrix are also in the form of a data table stored in the DataSet and returned to the client, along with the results.

The workflow of power flow online service platform is as follows: firstly, client browser chooses local raw data files and sends a computing request to the Web server; after receiving a request, in accordance with the principle of load balancing, Web server selects the current relatively idle WCF server, and redirects the browser webpage to the specific WCF server; on the WCF webpage, the users can upload data calculation, set calculation accuracy and call power flow calculation function to obtain the results. The structure and workflow of power flow online service platform is shown in Figure 2.

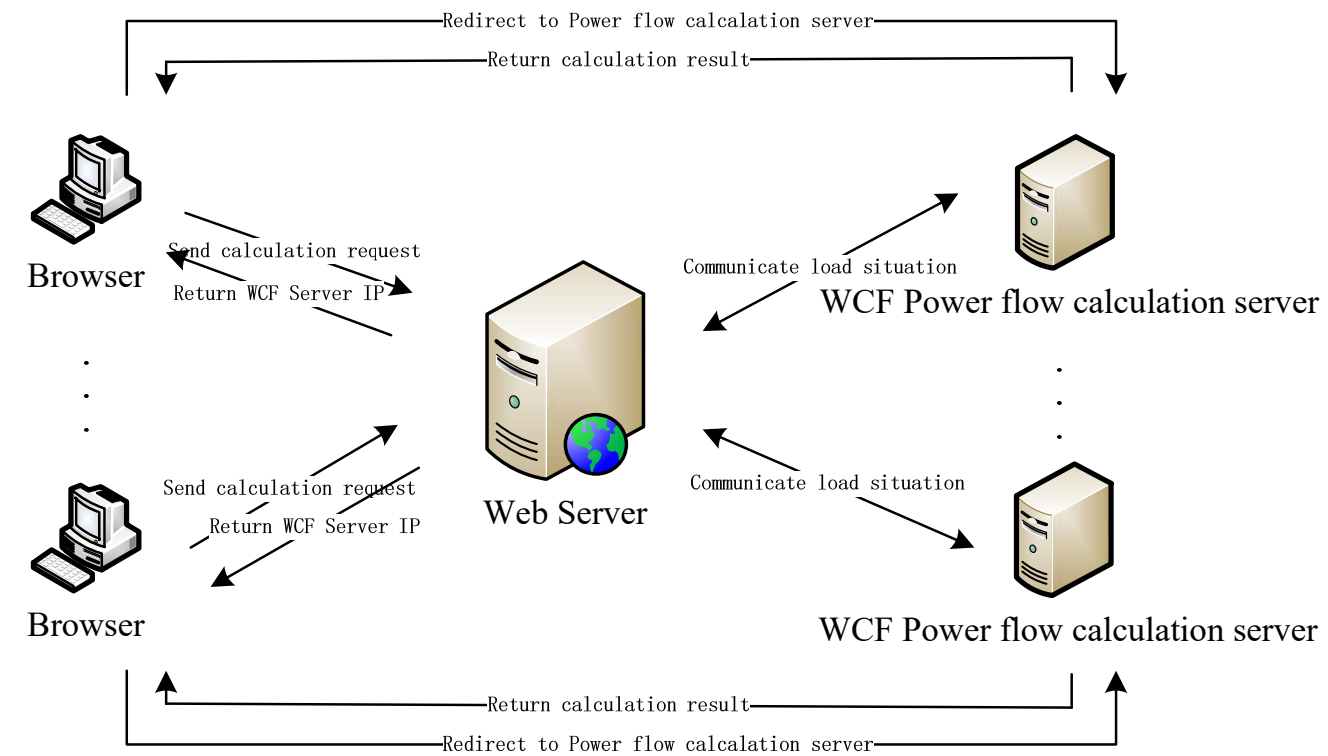

Fig. 2 The structure and workflow of power flow online service platform

The power flow online service platform is based on WCF, compiled by Microsoft Visual Studio 2010 and ASP.net, programmed by $\mathrm{C}$ \#. This program data source adopts from the IEEE standard power flow data. After verification, this platform is applicable to all IEEE standard power flow data, and the results of the calculation are in complete agreement with the results provided by IEEE.

\section{Multi-threading Management of Power Flow Calculation}

In order to meet the multi-user concurrent access, power flow online calculation service must ensure that the system has sufficient throughput. When a plurality of power flow calculation calls reach to the Web server, the server can make full use of the distributed computing resources, and the multi-threading process user's requests.

In order to deal with the concurrency problem of power flow calculation, the Web server sends the request of the client to the Queue when browser make flow calculation request through the function Enqueue. Detecting thread detects whether there is a request in queue circularly, if present, thread function removes and obtains the first record in queue by the function Dequeue. After obtaining record, Web servers starts thread, according to the load situation, chooses the relatively free WCF 
server, redirects the client browser webpage to the WCF server webpage, and destroy threads. The task in the queue is processed in order by a number of threads, which can achieve the power flow of multi-threading management. Power flow calculation queue and thread workflow is shown in Figure 3.

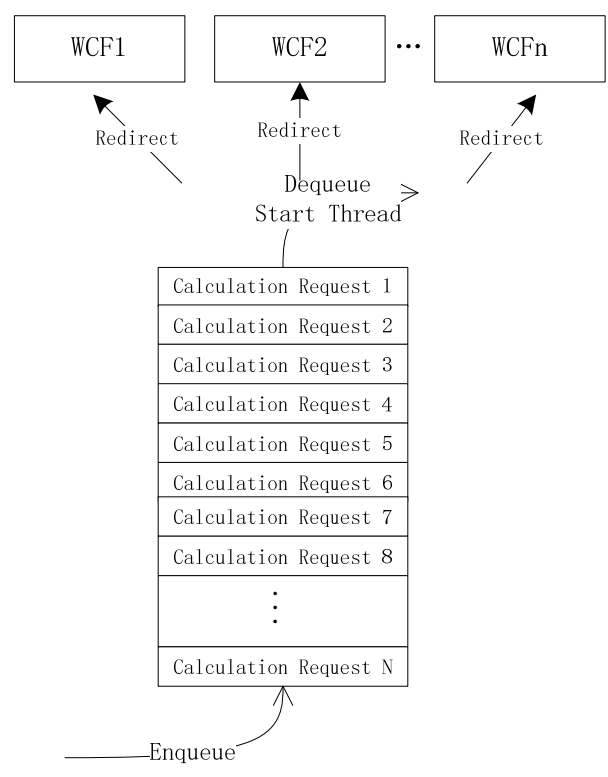

Fig. 3 Power flow calculation queue and thread workflow

\section{Conclusion}

This paper presents a design scheme of power flow calculation based on WCF technology, which provides two different service forms. Users can through the browser to view the service interface and instructions, and add the service interface to the local program, complete power flow calculation quickly; user can also access the website through the browser, upload the branch node raw data, figure out the calculation result online. This scheme utilizes the WCF technology advantages in distributed computing, and gives full play to the role of the Internet in optimization of computing resource, provides the ordinary users with open and free power flow calculation service. This scheme not only overcome power flow calculation's complex programming and other defects, and in future applications, due to the advantages of low coupling WCF technology, this method can realize power flow calculation algorithm of online upgrade and improvement, makes the flow calculation function more powerful, conforms to the era of the Internet development trend.

\section{References}

[1] Yan Shang, Zhangcan Huang, WCF: a new generation of Windows communication basic research and analysis [J]. Computer and digital engineering 2008,04:86-89.

[2] Zhe Dong, Management system of power quality monitoring equipment based on WCF [D]. North China Electric Power University, 2012

[3] Yejin Chen, The implementation of based data management system for power monitoring and control system base on WCF[D]. Southwest Jiao Tong University, 2013

[4] Bing Yan, Development of distributed application and based on WCF [J]. Computer knowledge and technology, 2008,19:68-70-74.

[5] Chengliang Wang, Ren Li, Zhuding Wang, Model of electric power distributed computing system based on service oriented architecture [J]. Journal of Chongqing University, 2011,02:69-73.

[6] Hong Long, Design and implementation of distributed workflow scheduling framework [D]. Tsinghua University, 2011. 